\title{
Optimierung eines miniaturisierten dynamischen Wärmestromdifferenzkalorimeters in LTCC-Technologie
}

\author{
Annica Brandenburg ${ }^{1}$, Jaroslaw Kita ${ }^{1}$, Eberhard Wappler ${ }^{2}$, Ralf Moos ${ }^{1}$ \\ ${ }^{1}$ Funktionsmaterialien, Universität Bayreuth, 95440 Bayreuth \\ funktionsmaterialien@uni-bayreuth.de \\ ${ }^{2}$ wsk Mess- und Datentechnik GmbH, 63450 Hanau
}

\begin{abstract}
Kurzfassung
In dieser Arbeit werden die Ergebnisse der Optimierungsarbeit einer miniaturisierten keramischen Messzelle für dynamische Wärmestromdifferenzkalorimetrie (engl. Heat Flux Differential Scanning Calorimetry, HFDSC) vorgestellt. Die DSC-Messzelle ist in Low Temperature Co-Fired CeramicsTechnologie (LTCC) hergestellt. Hierbei wird über Stapeln einzelner strukturierter keramischer Grünfolien ein komplexes dreidimensionales Bauteil erhalten. Dieses vereint alle Komponenten eines herkömmlichen DSC-Gerätes auf einem Chip mit den Abmessungen $11 \mathrm{~mm} \times 39 \mathrm{~mm} \times 1,4 \mathrm{~mm}$. In vorangehenden Arbeiten demonstrierten wir die Funktionalität der DSC-Messzelle - die gezeigten Ergebnisse wiesen eine gute Reproduzierbarkeit, sowie hohe Selektivität für analysierte Proben aus Indium, Zinn und Zink auf. Mit Fokus auf der Leistungsbedarfsreduzierung sowie der Homogenisierung der Temperaturverteilung im Probentiegel wurde ein simulationsgestützter Optimierungsprozess der keramischen Grundstruktur und der Heizergeometrie durchgeführt.
\end{abstract}

Key words: DSC, LTCC, Finite-Elemente-Methode

\section{Einführung}

In der dynamischen Wärmestromdifferenzkalorimetrie können Enthalpie änderungen sowie Wärmekapazitäten von Materialien detektiert werden [1]. Jedoch ist hierbei die Wahl der Materialien begrenzt, da während der thermischen Analyse aggressive oder korrosive Substanzen entstehen können, die die teuren DSC-Geräte kontaminieren oder gar beschädigen. In [2-5] präsentierten wir eine miniaturisierte DSC-Messzelle, die in LTCCTechnologie hergestellt ist und alle funktionellen Komponenten eines herkömmlichen DSC-Gerätes enthält. Die mit dieser Technologie einhergehende kostengünstige Herstellung der Messzellen macht einen Einweggebrauch und damit die Analyse aggressiver Proben möglich. Außerdem bietet sich aufgrund der geringen Größe des Systems und des geringen Leistungsbedarfes die Möglichkeit eines mobilen Einsatzes.

\section{Technologie}

Das Arbeitsprinzip der DSC-Messzelle basiert auf der Wärmestromdifferenzkalorimetrie. Hierbei werden eine Probe und eine inerte Referenzprobe in zwei Tiegeln platziert und einem definierten Temperatur-Zeit-Programm unterworfen. Treten Phasenübergänge in der Probe auf, ändert sich dessen Wärmekapazität, was zu einer Temperaturdifferenz $\Delta T$ zwischen Probe und Referenz führt. Diese wird über zwei in den Tiegeln integrierte Temperatursensoren detektiert. Mit Hilfe dieser Temperaturdifferenz kann der Wärmefluss unter Berücksichtigung des gerätespezifischen kalorischen Faktors (Gerätefaktor) berechnet werden. Er dient als Messsignal. Für weitere detaillierte Informationen wird der Leser auf einschlägige Literatur verwiesen, z.B. auf [1].

Die Anordnung der funktionellen Elemente in der DSC-Messzelle unterschiedet sich vom herkömmlichen Aufbau eines DSC-Gerätes: Probentiegel, Probentemperatursensor, Heizer, Referenztemperatursensor sowie Referenz liegen in vertikaler Anordnung im Messkopf der Zelle (siehe Abb. 1). 


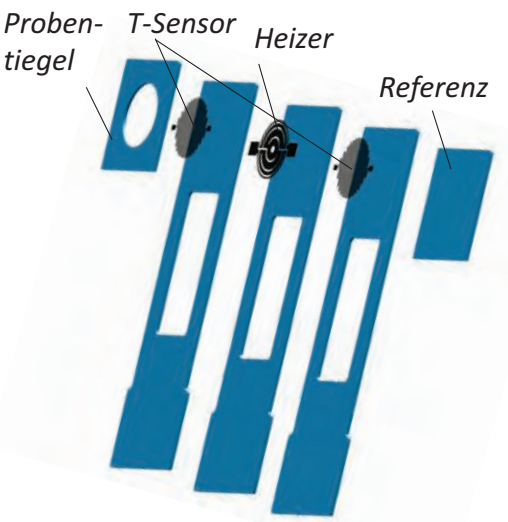

Abb. 1: Anordnung der funktionellen Elemente in der DSC-Messzelle.

Eine typische Messung eines IndiumSchmelzvorgangs in der DSC-Messzelle ist in Abb. 2 gezeigt.

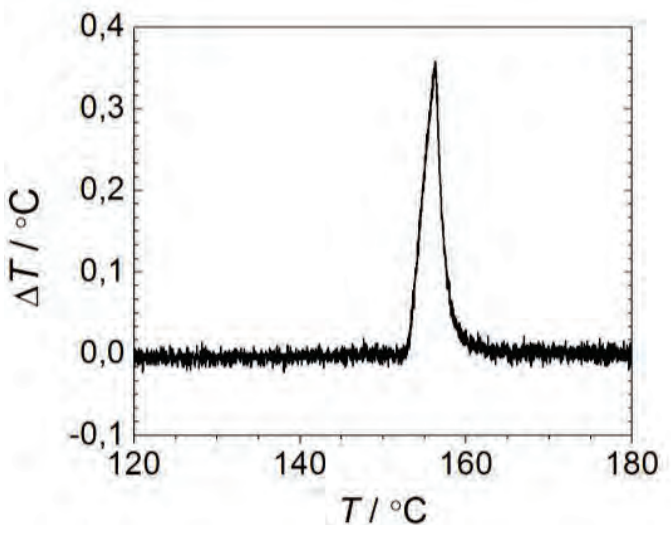

Abb. 2: Thermische Analysekurve von Indium $(10,993 \mathrm{mg})$ in der DSC-Messzelle, abgeändert nach nach [2].

\section{Optimierung der Struktur}

Simulationen physikalischer Effekte mit der Finite-Elemente-Methode (FEM) sind oft eine kosten- und zeitsparende Alternative zur klassischen Versuch-und-Irrtum-Vorgehensweise und werden mehr und mehr zum festen Bestandteil in technischen Entwicklungsprozessen. Für die Optimierung der DSCMesszelle führten wir eine simulationsgestützte Untersuchung vereinfachter Modelle durch, die aus der keramischen Grundstruktur, sowie einem im Messkopf integrierten Platinheizer aufgebaut ist. Die Simulationen wurden mit Hilfe der Simulationssoftware COMSOL Multiphysics in mehreren Schleifen durchgeführt. Als Randbedingungen wurden konvektive Kühlung sowie Strahlungsverluste an allen Außenflächen definiert.

\section{Reduzierung des Leistungsbedarfs}

Um die thermische Masse der DSC-Messzelle zu verringern und damit ihren Leistungsbedarf weiter zu reduzieren, wurden Bereiche im Messkopf, in denen sich keine funktionellen Elemente befinden und die daher keinen Einfluss auf die Funktionsweise der Messzelle haben, reduziert. Auf diese Weise wurde die Masse des Chipkopfes um ca. $45 \%$ verringert. Zusätzlich wurde die Breite der Stege verringert, um den Wärmefluss aus dem Messzellenkopf zu minimieren. Abb. 3 zeigt die simulierten Temperaturverteilungen beider Strukturen bei einer Maximaltemperatur von $225^{\circ} \mathrm{C}$. Durch die Verringerung der Masse im beheizten Bereich und die schmaleren Stege konnte der Leistungsbedarf der optimierten Struktur um $42 \%(1,9 \mathrm{~W}$ auf $1,1 \mathrm{~W})$ gegenüber der Ausgangsstruktur verringert werden.

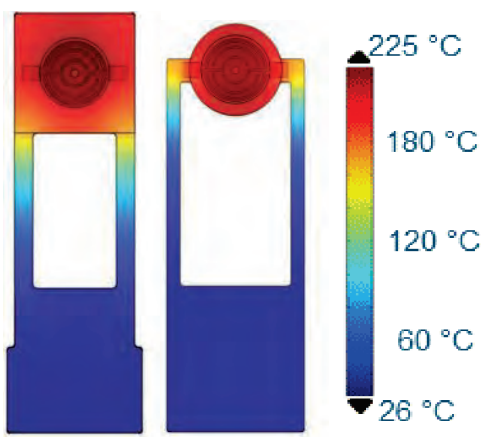

Abb. 3: Temperaturverteilung der Ausgangsstruktur und der optimierten DSC-Messzelle mit verringertem Messkopfvolumen. Die Modelle sind transparent dargestellt, um die Position des Heizers zu verdeutlichen.

\section{Homogenisierung des Temperaturprofils}

Eine homogene Probentemperatur ist essentiell für die exakte Bestimmung von Phasenübergängen sowie für die Detektion eng benachbarter kalorischer Effekte. Hierdurch wird gewährleistet, dass z.B. dicht nebeneinander liegende Phasenübergänge zweier Substanzen in einem Gemenge jeweils zeitlich konzentriert verlaufen und somit schmale Signalpeaks erhalten werden.

Der Heizer der Ausgangsstruktur war so konstruiert, dass er eine konstante Heizleistung pro Fläche erzeugt. Wie in Abb. 4 a) zu sehen ist, tritt jedoch sowohl durch den Wärmeverlust über die Stege als auch durch erhöhte konvektive Wärmeverluste an den Außenrändern eine verringerte Temperatur im äußeren Tiegelbereich auf. Um die Homogenität der Temperaturverteilung im Tiegel zu erhöhen, wurden in einem zweiten Modellierungsschritt die Breite der Heizleiter, 
sowie die Abstände zwischen diesen variiert. Zusätzlich wurden Heizmäander zwischen dem Messkopf und den Stegen eingeführt, um den erhöhten Wärmefluss zu den Stegen zu kompensieren.

Zur Quantifizierung der Homogenität des Temperaturprofiles wurde die mittlere Temperaturabweichung im Tiegel, $d_{T}$, herangezogen:

$$
d_{T}=\frac{\Sigma\left|T_{i}-T_{m}\right|}{n}
$$

wobei $T_{i}$ die lokale Temperatur am Punkt $i$,

$T_{m}$ die mittlere Temperatur im Tiegel und

$n$ die Anzahl der Messpunkte

darstellen.

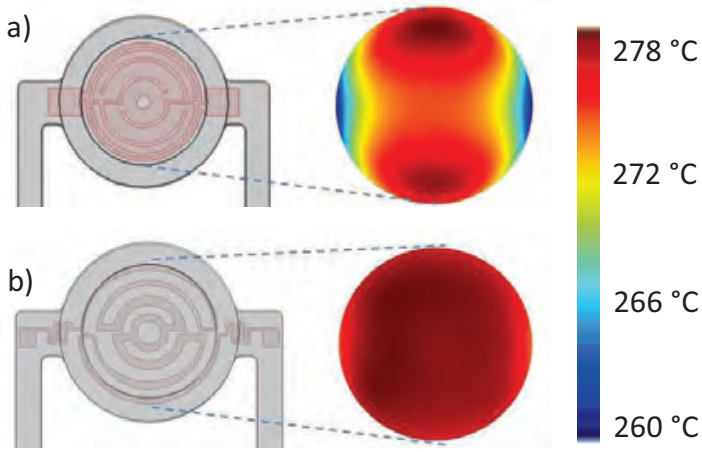

Abb. 4: Temperaturverteilung im Messtiegel der a) Ausgangsstruktur und b) der Struktur mit optimierter Heizergeometrie bei einer Maximaltemperatur von $278{ }^{\circ} \mathrm{C}$.

Wie in Abb. 4 b) zu sehen ist, kann durch eine Anpassung der Heizleiterbreite und des Abstandes zwischen den Heizleitern eine gleichmäßigere Temperaturverteilung im Tiegelbereich erzeugt werden. Die mittlere Temperaturabweichung konnte hierdurch bei einer Maximaltemperatur von $278{ }^{\circ} \mathrm{C}$ von $2,78{ }^{\circ} \mathrm{C}$ auf $0,58{ }^{\circ} \mathrm{C}$ gesenkt werden.

\section{Zusammenfassung und Ausblick}

Ein simulationsgestützter Optimierungsprozess einer neuartigen miniaturisierten keramischen DSC-Messzelle zeigt vielversprechende Ergebnisse hinsichtlich der Verringerung ihres Leistungsbedarfes, sowie einer homogenen Temperaturverteilung im Probentiegel. Durch die Reduzierung der thermischen Masse im Messkopfbereich sowie durch das Einführen schmalerer Stege konnte zunächst der Leistungsbedarf um ca. $42 \%$ reduziert werden.

In einem zweiten Optimierungsschritt wurde die Heizergeometrie neu konstruiert. Durch das Anpassen der Heizleiterbreite und des Abstandes zwischen den Heizleitern sowie durch die Einführung zusätzlicher Heizwendel zwischen Tiegel und Stegen, konnte das Temperaturprofil homogenisiert werden. Die mittlere Temperaturabweichung wurde, bei einer Maximaltemperatur von $278{ }^{\circ} \mathrm{C}$, von $2,78{ }^{\circ} \mathrm{C}$ auf $0,58{ }^{\circ} \mathrm{C}$ reduziert. Hierdurch können wir von einer deutlich höheren Trennschärfe unserer DSC-Messzelle ausgehen.

Derzeit arbeiten wir an der Charakterisierung eines neuen Prototypen, der nach den Abmessungen des optimierten Modelles gefertigt ist (siehe Abb. 5). Zur Validierung der Simulationsergebnisse werden Infrarotaufnahmen während des Aufheizens angefertigt sowie der Einfluss der gezeigten strukturellen Maßnahmen auf die Messcharakteristik untersucht.
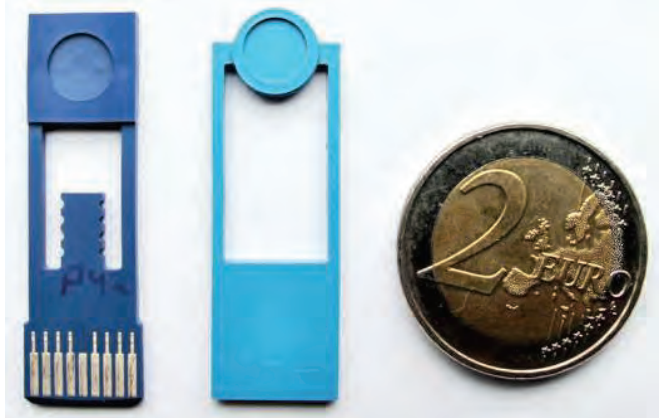

Abb. 5: Foto der Ausgangsstruktur und der optimierten DSC-Messzelle im Größenvergleich mit

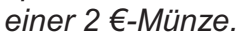

\section{Danksagung}

Die vorgestellten Ergebnisse sind im Rahmen eines Projektes entstanden, das durch das Zentrale Innovationsprogramm Mittelstand (KF2116719WM2) gefördert wurde.

\section{Literatur}

[1] G.W.H. Höhne, W.F. Hemminger, H.-J. Flammersheim, "Differential Scanning Calorimetry", 2nd ed., Springer, Berlin, 2003

[2] W. Missal, J. Kita, E. Wappler, F. Bechtold, R. Moos, "Calorimetric sensitivity and thermal resolution of a novel miniaturized ceramic DSC chip in LTCC technology", Thermochim. Acta 543 (2012) 142-149, doi:10.1016/j.tca.2012.05.019

[3] R. Moos, W. Missal, J. Kita, E. Wappler, F. Gora, A. Kipka, T. Bartnitzek, F. Bechtold, D. Schabbel, B. Pawlowski: Einweg-DSC-Chip Sensor 2011, fms-Sondersession 2011, Workshop Sensorforschung für Medizin und Technik. Ergebnisse aus der industriellen Gemeinschaftsforschung, im Rahmen der Sensor + Test 2011, 9. Juni 2011, Nürnberg, p. 4-9. 
[4] W. Missal, J.Kita, E. Wappler, F. Gora, A. Kipka, T. Bartnitzek, F. Bechtold, D. Schabbel, B. Pawlowski, R. Moos, "Miniaturized ceramic differential scanning calorimeter with integrated oven and crucible in LTCC technology", Sens. Actuators A 172 (2011) 21-26, doi: 10.1016/j.sna.2011.01.025

[5] J. Kita, W. Missal, E. Wappler, F. Bechtold, R. Moos, "Development of a Miniaturized Ceramic Differential Calorimeter Device in LTCC

Technology", J. Ceram. Sci. Tech., in press, doi: 10.4416/JCST00008-2013 